\title{
Avaliação da atividade moluscicida de extratos vegetais sobre Achatina fulica Bowdich (Mollusca, Achatinidae)
}

\author{
Evaluation of the molluscicidal activity of vegetal extracts on \\ Achatina fulica Bowdich (Achatinidae)
}

\author{
Licielo Romero Vieira', Grasielle Soares Gusman², Silvane Vestena1*

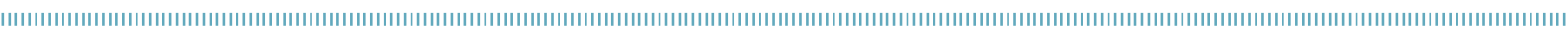

RESUMO: Achatina fulica Bowdich (Achatinidae) é um gastrópode terrestre pulmonado e tornou-se praga de pequenos cultivos de subsistência, sendo considerado uma ameaça à agricultura sustentável em alguns países, demandando altos custos em tentativas de controle, as quais, em sua maioria, resultaram em fracasso; ainda, pode atuar como hospedeiro intermediário de nematódeos. Nesse contexto, a procura por substâncias facilmente biodegradáveis tem aumentado o interesse pelo uso de moluscicidas de origem vegetal. Assim, o estudo teve como objetivo verificar a ação de extratos aquosos de folhas de Bacharis dracunculifolia DC., Morus rubra L., Cyperus rotundus L. e Euphorbia heterophylla L. e gemas florais de Sygygium aromaticum (L.) Merr. \& Perry como possíveis moluscicidas. Destas, $S$. aromaticum e E. heterophylla foram ativas como moluscicidas, e $C$. rotundus náo exibiu atividade moluscicida sobre esse gastrópode. Independentemente da espécie vegetal testada, nenhuma demonstrou atividade ovicida. Os resultados do presente estudo indicam que os extratos aquosos de folhas de E. heterophylla e de gemas florais de $S$. aromaticum podem se constituir em uma estratégia viável de controle alternativo de moluscos terrestres.

PALAVRAS-CHAVE: Achatina fulica Bowdich.; gastrópode terrestre; extrato vegetal.
ABSTRACT: Achatina fulica Bowdich (Achatinidae) is a terrestrial pulmonary gastropod that has become a pest in small subsistence plantations. Also it is considered as a threat to sustainable agriculture in some countries, which demand high investments in order to control it; however, the most part of the tried methods have failed. This species can also act as an intermediate host of nematodes. In this context, the search for compounds easily biodegradable has increased the interest for using molluscicidal agents obtained from plant sources. Therefore, this study aimed to evaluate leaves extracts of Bacharis dracunculifolia DC., Morus rubra L., Cyperus rotundus L. and buds extracts of Euphorbia heterophylla L. and Sygygium aromaticum (L.) Merr. \& Perry as possible molluscicidal agents. From these species, S. aromaticum and E. heterophylla were active as molluscicidal agents affecting survival rate of $A$. fulica and, $C$. rotundus did not show molluscicidal activity over this gastropod. None of the plant species tested showed ovicidal activity. The results of the present study indicate that the aqueous extracts of $S$. aromaticum buds and $E$. heterophylla leaves can be a viable approach for alternative control of terrestrial molluscs.

KEYWORDS: Achatina fulica Bowdich; terrestrial gastropod; plant extract. 


\section{INTRODUÇÃO}

O caramujo exótico invasor Achatina fulica Bowdich (caramujo africano) é nativo do leste da África, onde sua distribuição original tem limite ao sul, em Natal (África do Sul), e ao norte, na Somália. Atualmente, devido ao seu sucesso como invasor, sua distribuição abrange quase todos os continentes, inclusive regiōes temperadas (RAUT; BARKER, 2002). No Brasil, foi introduzido na década de 1980 (Telles et al., 1997), em uma tentativa de substituição de cultivo do "escargot" (Helix aspersa Müller). A espécie africana surgiu como uma boa opção aos criadores por apresentar vantagens, como a maior adaptação ao clima dos trópicos, facilitando a criaçáo e a reduçáo no tempo de crescimento individual e populacional, resultando em maior produção. A perda de interesse na criação, motivada principalmente pelo baixo retorno financeiro e pelas dificuldades para escoação da produção, levou alguns criadores a abandonarem exemplares no ambiente, acarretando sua rápida dispersão pelo território brasileiro (ZANOL et al., 2010).

Essa espécie pode transmitir doenças, causar danos ao meio ambiente e prejuízos econômicos. Esses danos são geralmente elevados devido ao comportamento gregário e às frequentes explosôes populacionais da espécie. Tal infestação aumenta de importância quando consideramos que esse caramujo é hospedeiro intermediário de larvas de nematódeos Metastrogyloidea parasitos do ser humano, bem como de felídeos, cães e outros mamíferos. O nematódeo Angiostrongylus cantonensis, agente etiológico da meningite eosinofílica, pode ter também $A$. fulica como um dos seus principais hospedeiros (BARbosa et al., 2002; Thiengo et al., 2007). Adicionalmente, $A$. fulica pode atuar como praga agrícola ou levar à perda da biodiversidade local (CowiE; RoBinson, 2003), em razáo do seu potencial competitivo, predatório (Meyer et al., 2008), ou de transmissão de doenças para animais selvagens (Thiengo et al., 2008). Recentemente, Colley; FIsCher (2009) relataram impactos à biodiversidade causados pela infestaçáo de $A$. fulica, tanto decorrentes da competição por espaço e alimento com os moluscos nativos quanto pela interferência na cadeia alimentar.

Atualmente, existem várias substâncias consideradas potentes moluscicidas, mas sua ação biocida, seu alto custo e a dificuldade de seu transporte para distantes áreas rurais tornam necessário o investimento em novas pesquisas, em busca de alternativas mais bem-sucedidas de controle malacológico (Pinheiro; Cortez, 2003; Leyton et al., 2005; Singh et al., 2012). A necessidade de moluscicidas eficientes e ecologicamente aceitáveis acompanhou a tendência de se pesquisar plantas na busca de substâncias ativas para o desenvolvimento de produtos alternativos. Adicionalmente, a utilização de extratos vegetais no controle natural desperta atençáo pelos excelentes resultados obtidos sobre moluscos aquáticos (YAdAV; SINGH, 2001; SINGH et al., 2012). Contudo, estudos sobre a atividade de plantas moluscicidas em moluscos terrestres são pouco comuns, apesar de sua grande importância parasitológica e agrícola. As semelhanças anatomofisiológicas entre espécies de moluscos aquáticos e terrestres sugerem que estratégias de controle químico possam ter eficiência semelhante para os dois grupos de invertebrados (Pinheiro; Cortez, 2003; Afonso-Neto et al., 2010; ZANOL et al., 2010).

Assim, a procura por substâncias facilmente biodegradáveis tem aumentado o interesse pelo uso de moluscicidas de origem vegetal; sendo assim, o presente estudo teve como objetivo avaliar a atividade moluscicida de extratos vegetais de alecrim-do-campo (Bacharis dracunculifolia DC.), amora (Morus rubra L.), tiririca (Cyperus rotundus L.), leiteira (Euphorbia heterophylla L.) e cravo-da-índia (Sygygium aromaticum (L.) Merr. \& Perry) sobre o gastrópode terrestre A. fulica.

\section{MATERIAL E MÉTODOS}

Para a realização dos experimentos, foram utilizadas folhas adultas de B. dracunculifolia, de M. rubra, de E. heterophylla e de C. rotundus, obtidas no município de Muriaé (MG), e gemas florais de S. aromaticum, obtidas no comércio local, sendo que as folhas foram secas em estufa a $40^{\circ} \mathrm{C}$ até a obtenção de massa seca constante.

Para a obtenção do extrato aquoso das espécies em estudo, foram utilizadas folhas secas e gemas florais na concentração de $1 \mathrm{~g} 10 \mathrm{~mL}^{-1}$ (peso/volume) e trituradas em um moinho tipo Willey. A mistura foi deixada em repouso por $48 \mathrm{~h}$ na geladeira $\left(5 \pm 1^{\circ} \mathrm{C}\right)$, sendo, após, filtrada em funil de Büchner, por 2 vezes, usando-se papel-filtro qualitativo. $\mathrm{O}$ extrato aquoso foi diluído em 5 concentraçôes diferentes (10, 30, 50, 70 e $100 \%)$ e utilizou-se água destilada como tratamento controle, sendo que para a concentraçáo de $100 \%$ foi utilizado o "extrato puro".

Foram utilizados 20 ovos após a oviposição e 10 moluscos após 2 dias de eclosão em 3 repetiçôes. O experimento com os ovos e com os moluscos foi pulverizado a cada 2 dias com $5 \mathrm{~mL}$ (20 borrifadas) dos extratos aquosos das espécies vegetais testadas. O experimento foi mantido por um período de 30 dias. Foram obtidas as taxas de eclosão e de sobrevivência e a massa final (NASCIMENTO et al., 2006).

O delineamento utilizado foi inteiramente casualizado para todos os parâmetros analisados, os resultados foram submetidos à análise de variância (ANOVA), e as médias, comparadas pelo teste Tukey a $5 \%$ de probabilidade (Beiguelman, 2002).

\section{RESULTADOS E DISCUSSÃO}

Verificou-se que os extratos aquosos das quatro espécies testadas não interferiram significativamente na eclosão dos ovos de 
A. fulica, já que foi observado em todos os tratamentos elevado percentual de eclosão, independentemente da concentração dos extratos utilizados (Tabela 1).

Tabela 1. Eclosão, sobrevivência e massa final de Achatina fulica sob efeito de extratos aquosos de alecrim-do-campo (Baccharis dracunculifolia DC.), tiririca (Cyperus rotundus L.), amora (Morus rubra L.), cravo-da-índia (Syzygium aromaticum (L.) Merr. \& Perry) e leiteira (Euphorbia heterophylla L.). Muriaé, 2013.

\begin{tabular}{|c|c|c|c|}
\hline $\begin{array}{l}\text { Conc. } \\
(\%)\end{array}$ & $\begin{array}{c}\text { Eclosão } \\
\qquad \%)\end{array}$ & $\begin{array}{c}\text { Sobrevivência } \\
\text { (\%) }\end{array}$ & $\begin{array}{c}\text { Massa final } \\
\text { (g) }\end{array}$ \\
\hline \multicolumn{4}{|c|}{ Baccharis dracunculifoia } \\
\hline 0 & $91,0 \pm 0,27 a$ & $81,9 \pm 0,95 a$ & $1,565 \pm 0,17 a$ \\
\hline 10 & $87,5 \pm 0,10 a$ & $86,0 \pm 0,67 a$ & $1,358 \pm 0,23 a$ \\
\hline 30 & $85,5 \pm 0,35 a$ & $82,5 \pm 0,22 \mathrm{a}$ & $1,090 \pm 0,08 \mathrm{ab}$ \\
\hline 50 & $90,5 \pm 0,25 a$ & $81,6 \pm 0,64 a$ & $1,108 \pm 0,06 \mathrm{ab}$ \\
\hline 70 & $91,5 \pm 0,30 a$ & $83,3 \pm 0,55 a$ & $0,735 \pm 0,03 b$ \\
\hline 100 & $87,5 \pm 0,24 a$ & $76,5 \pm 0,78 a$ & $0,765 \pm 0,05 b$ \\
\hline \multicolumn{4}{|c|}{ Cyperus rotundus } \\
\hline 0 & $96,0 \pm 0,10 a$ & $93,5 \pm 0,65 a$ & $1,895 \pm 0,37 a$ \\
\hline 10 & $94,5 \pm 0,45 a$ & $86,5 \pm 0,67 a$ & $1,752 \pm 0,28 a$ \\
\hline 30 & $94,0 \pm 0,75 a$ & $91,0 \pm 0,12 a$ & $1,632 \pm 0,36 a$ \\
\hline 50 & $95,8 \pm 0,25 a$ & $88,5 \pm 0,56 a$ & $1,502 \pm 0,95 a$ \\
\hline 70 & $91,3 \pm 0,36 a$ & $87,2 \pm 0,15 a$ & $1,320 \pm 0,65 a$ \\
\hline 100 & $92,5 \pm 0,30 a$ & $91,5 \pm 0,30 a$ & $1,462 \pm 0,74 a$ \\
\hline
\end{tabular}

\section{Morus rubra}

\begin{tabular}{cccc}
\hline 0 & $90,0 \pm 0,10 a$ & $71,9 \pm 0,85 a$ & $1,166 \pm 0,17 \mathrm{a}$ \\
\hline 10 & $83,3 \pm 0,45 \mathrm{a}$ & $76,0 \pm 0,37 \mathrm{a}$ & $1,159 \pm 0,23 \mathrm{a}$ \\
\hline 30 & $90,0 \pm 0,75 \mathrm{a}$ & $72,9 \pm 0,12 \mathrm{a}$ & $1,160 \pm 0,08 \mathrm{a}$ \\
\hline 50 & $86,6 \pm 0,25 \mathrm{a}$ & $71,5 \pm 0,08 \mathrm{a}$ & $0,855 \pm 0,06 \mathrm{ab}$ \\
\hline 70 & $83,3 \pm 0,36 \mathrm{a}$ & $80,0 \pm 0,05 \mathrm{a}$ & $0,662 \pm 0,03 \mathrm{~b}$ \\
\hline 100 & $80,0 \pm 0,20 \mathrm{a}$ & $79,1 \pm 0,10 \mathrm{a}$ & $0,145 \pm 0,05 \mathrm{c}$ \\
\hline Syzygium aromaticum & & \\
\hline 0 & $91,0 \pm 0,10 \mathrm{a}$ & $80,0 \pm 0,85 \mathrm{a}$ & $1,786 \pm 0,27 \mathrm{a}$ \\
\hline 10 & $87,5 \pm 0,45 \mathrm{a}$ & $73,5 \pm 0,37 \mathrm{a}$ & $1,159 \pm 0,23 \mathrm{ab}$ \\
\hline 30 & $85,0 \pm 0,75 \mathrm{a}$ & $63,0 \pm 0,12 \mathrm{ab}$ & $1,040 \pm 0,08 \mathrm{~b}$ \\
\hline 50 & $86,6 \pm 0,25 \mathrm{a}$ & $58,5 \pm 0,15 \mathrm{~b}$ & $0,785 \pm 0,20 \mathrm{c}$ \\
\hline 70 & $85,5 \pm 0,36 \mathrm{a}$ & $45,5 \pm 0,23 \mathrm{c}$ & $0,352 \pm 0,08 \mathrm{~d}$ \\
\hline 100 & $89,0 \pm 0,10 \mathrm{a}$ & $40,0 \pm 0,17 \mathrm{c}$ & $0,105 \pm 0,05 \mathrm{e}$ \\
\hline Euphorbia heterophylla & & \\
\hline 0 & $88,0 \pm 0,25 \mathrm{a}$ & $82,0 \pm 0,25 \mathrm{a}$ & $1,325 \pm 0,27 \mathrm{a}$ \\
\hline 10 & $87,5 \pm 0,36 \mathrm{a}$ & $83,5 \pm 0,17 \mathrm{a}$ & $1,268 \pm 0,23 \mathrm{a}$ \\
\hline 30 & $83,0 \pm 0,65 \mathrm{a}$ & $81,0 \pm 0,22 \mathrm{ab}$ & $1,015 \pm 0,08 \mathrm{ab}$ \\
\hline 50 & $84,6 \pm 0,75 \mathrm{a}$ & $68,5 \pm 0,36 \mathrm{~b}$ & $0,979 \pm 0,20 \mathrm{~b}$ \\
\hline 70 & $80,5 \pm 0,86 \mathrm{a}$ & $55,5 \pm 0,55 \mathrm{bc}$ & $0,569 \pm 0,08 \mathrm{c}$ \\
\hline 100 & $87,0 \pm 0,90 \mathrm{a}$ & $42,5 \pm 0,28 \mathrm{c}$ & $0,463 \pm 0,05 \mathrm{c}$ \\
\hline
\end{tabular}

Médias \pm DP seguidas pela mesma letra nas colunas não diferem significativamente pelo teste Tukey a $5 \%$ de probabilidade.
A taxa de sobrevivência de indivíduos de $A$. fulica também não foi afetada significativamente quando foram utilizados os extratos aquosos das espécies vegetais, exceto quando foram utilizados extratos de $S$. aromaticum e de E. heterophylla; ainda, com esses dois últimos extratos vegetais, o percentual de sobrevivência diminuiu com o aumento das concentraçôes utilizadas, sendo reduzido a partir da concentraçấo de 50\%, quando comparado ao tratamento controle. Também, para ambos os extratos vegetais, quando foi utilizado o extrato puro (100\%), ocorreu diminuiçẫo de 50\% na taxa de sobrevivência, quando comparado ao tratamento controle (Tabela 1).

Em relaçáo à oviposição, observou-se que a massa final dos indivíduos de $A$. fulica apresentou diferenças estatísticas quando foram utilizados os extratos de B. dracunculifolia, de E. heterophylla, de $S$. aromaticum e de $M$. rubra, redução essa verificada a partir da concentração de $50 \%$ para $E$. heterophylla e $S$. aromaticum e a partir de $70 \%$ para B. dracunculifolia e M. rubra; ainda, como observado com as taxas de eclosão e de sobrevivência, quando foram utilizados extratos aquosos de C. rotundus, não foram observadas diferenças estatísticas para a massa final, denotando que não é adequado indicar essa espécie vegetal no controle biológico desse gastrópode terrestre (Tabela 1). Nota-se que, entre as três espécies vegetais com possível atividade moluscicida, o extrato vegetal de $S$. aromaticum se mostrou o mais efetivo para a utilização no controle desse gastrópode terrestre, reduzindo a massa final a partir da concentração de $30 \%$, quando comparado ao tratamento controle; já na concentração de $50 \%$ esse parâmetro foi reduzido pela metade, assim como quando utilizado o extrato aquoso puro a reduçáo foi de 17 vezes, quando comparado com o tratamento controle (Tabela 1).

Alguns pesquisadores justificam a não atuação de extratos vegetais de espécies da família Cyperaceae pela ausência de efeito tóxico e/ou da classe do metabólito secundário presente nas partes vegetais e/ou pelas diferenças na sensibilidade desses invertebrados às toxinas e/ou aos fatores ambientais, como a insolação, que podem modular diferenças químicas ou metabólicas entre as espécies dessas famílias e ter diferentes impactos sobre a fisiologia da espécie de molusco testada. A análise desse conjunto de fatores pode nortear a seleção de plantas para estudos de atividade moluscicida, favorecer o delineamento dos ensaios experimentais e contribuir para a compreensão dos mecanismos tóxicos das substâncias envolvidas.

Entretanto, Ruiz et al. (2005), testando Eleocharis, também da família Cyperaceae, como moluscicida sobre Biomphalaria glabrata Say, relataram que extratos obtidos de espécies desse gênero são submetidos a ensaios de atividade moluscicida e de toxicidade, sendo ativos contra a desova de B. glabrata. Ainda, é abordado por MARSTOn et al. (1996) que, dentro da família Cyperaceae, foi encontrado apenas o relato de atividade moluscicida dos extratos de $C$. luzulae, também contra caramujos adultos B. glabrata.

Dentre as várias espécies vegetais com atividade moluscicida, estão espécies da família Euphorbiaceae (Euphorbia cotinifolia L., 
E. milii des Moul. var. splendens (Bojer ex Hook) Ursch \& Leandri e E. tirucalli L.) e, como no estudo em questão, E. heterophylla; não foi verificado que essa espécie seja considerada ovicida, mas foi ativa reduzindo o crescimento dos indivíduos de $A$. fulica, conforme observado na taxa de sobrevivência e na massa final (Tabela 1).

Afonso-Neto et al. (2010), avaliando a atividade moluscicida do látex das três espécies de Euphorbia citadas anteriormente, E. cotinifolia, E. milii var. splendens e E. tirucalli, sobre Leptinaria unilamellata D'Orbigny, verificaram que apenas E. milii var. splendens apresentou efeito moluscicida sobre L. unilamellata, indicando que o látex dessa espécie pode se constituir em uma estratégia viável de controle natural de moluscos terrestres e que, embora citadas na literatura como tóxicas para moluscos aquáticos, $E$. cotinifolia e E. tirucalli não exibiram atividade moluscicida sobre L. unilamellata.

YADAV; Singh (2011), também utilizando uma espécie de Euphorbiaceae, E. hirta Linn, abordaram que o látex dessa espécie, assim como outros compostos vegetais moluscicidas, foi avaliado em sua ação sobre caramujos de água doce Lymnaea (Radix) acuminata e Indoplanorbis exustus em represas, mostrando seu efeito letal sobre as duas espécies de caramujo e para peixes de água doce Channa punctatus Bloch.

Muitas são as espécies de plantas tropicais apontadas na literatura como possuidoras de substâncias moluscicidas. Podem ser citadas espécies das famílias Asteraceae, Euphorbiaceae, Fabaceae, Phytolacaceae, Sapindaceae e Solanaceae. Tão diversos como as espécies que as produzem são os produtos naturais moluscicidas. Dentre essas substâncias tóxicas para moluscos, encontram-se alcaloides, saponinas triterpenoides, sesquiterpenoides e derivados fenólicos (Marston; Hostettman, 1985; Rug; Ruppel, 2000; Al-Zanbagi et al., 2001; Santos et al., 2009). Na família Euphorbiaceae, plantas lactescentes ricas em terpenoides com representantes nativos e exóticos (ZANI et al., 1993; Mabberley, 1997; Oliveira; Akisue, 1997), o gênero Euphorbia teve os látices de algumas espécies testados em formulaçóes e concentraçóes variadas, apresentando atividade moluscicida excepcional em gastrópodes pulmonados aquáticos (Pereira et al., 1978; Al-Zanbagi et al., 2001), mas ainda sáo poucas as pesquisas a respeito de controle de moluscos gastrópodes terrestres, especialmente com extratos vegetais (Afonso-Neto et al., 2010).

Como representante da família Myrtaceae está o cravo-da-índia (S. aromaticum), uma possibilidade de ser utilizado com propriedades moluscicidas, sendo que a gema floral seca do cravo-da-índia é usada principalmente como condimento na culinária, devido ao seu aroma e sabor marcantes, conferido por um composto fenólico volátil, o eugenol (RAINA et al., 2001; Oliveira et al., 2007; Agra et al., 2008). Segundo GobboNeto; Lopes (2008) e Oliveira et al. (2008), o eugenol pode ser utilizado como anti-inflamatório, anestésico, antisséptico, antioxidante, alelopático e repelente. Adicionalmente, o eugenol é muito usado na Odontologia como componente de seladores e de outros produtos antissépticos de higiene bucal, tendo comprovado efeito bactericida, e alguns trabalhos mostraram que o eugenol ou os extratos de $S$. aromaticum apresentam atividade nematicida, inseticida, antiviral e fungicida (TsAO; Yu, 2000; Nascimento et al., 2006).

Além disso, recentemente, em trabalhos desenvolvidos no Amazonas, no Instituto Nacional de Pesquisa da Amazônia (INPA), foi elucidada uma metodologia de utilização de extratos aquosos de gemas florais de cravo-da-índia no controle de larvas do mosquito da dengue (Aedes aegypti L.), relatando que, em laboratório, as larvas morrem em 24 h quando na presença do extrato aquoso pela presença do eugenol como substância ativa presente nas partes florais, sendo, assim, uma possibilidade de testar seu efeito como moluscicida natural, já observado no presente estudo com $A$. fulica.

Colley (2010) trabalhou com A. fulica no estado do Rio de Janeiro e, depois de avaliar as medidas de controle dessa espécie existentes (controle biológico, químico e físico), relatou que a primeira etapa do manejo é diagnosticar o perfil de ocupação do invasor, para escolher posteriormente a melhor medida a ser utilizada e adaptá-la à realidade local. Ainda, relata que alguns municípios desse Estado têm feito uso de moluscicidas (produtos à base de metaldeído) no controle desse caramujo. Porém, tais produtos não são recomendados, pela sua toxicidade e por não serem específicos para $A$. fulica, matando também outras espécies de moluscos. Além disso, tais produtos são danosos a outros organismos, como pássaros e outros predadores, que se alimentam do veneno ou do caramujo envenenado (BARKER; WATTS, 2002).

A rápida ampliação da distribuição de $A$. fulica na região chama mais uma vez a atenção para a importância de planos de manejo e controle dessa espécie. É importante que esses planos sejam contínuos e generalizados em todos os municípios, uma vez que a reinfestação ocorre de maneira acelerada (Simiâo; FisCHER, 2004). Municípios que ainda não registraram a presença de $A$. fulica deveriam investir em medidas preventivas, como campanhas de conscientização, para que a população local não transporte esse caramujo e seja capaz de detectar a introdução desse molusco em sua fase inicial, facilitando consideravelmente o controle da invasão. Considerando o nível atual de infestação de Minas Gerais, a erradicação de $A$. fulica é pouco provável, requerendo um grande investimento econômico. Entretanto, medidas de controle que mantenham as populaçóes de $A$. fulica em níveis toleráveis são factíveis, além de extremamente necessárias, haja vista que moluscicidas sintéticos apresentam limitaçôes técnicas que estimulam a busca por substitutos naturais.

\section{CONCLUSÃO}

Todos os extratos vegetais testados não demonstram atividade ovicida; entretanto, todos eles são ativos como moluscicidas, reduzindo a sobrevivência e a massa final do gastrópode terrestre A. fulica, exceto quando são utilizados extratos de C. rotundus. 
AGRA, M.F.; SILVA, K.N.; BASÍLIO, I.J.L.D.; FRANÇA, P.F.; BARBOSAFILHO, J.M. Survey of medicinal plants used in the region Northeast of Brazil. Revista Brasileira de Farmacognosia, v.18, p.472-508, 2008.

AL-ZANBAGI, N.A.; BARRET, J.; BANAJA, A.A. Laboratory evaluation of the molluscicidal properties of some Saudi-Arabian Euphorbiales against Biomphalaria pfeifferi. Acta Tropica, v.78, p.23-29, 2001.

AFONSO-NETO, I.S.; BESSA, E.A.; SOARES, G.L.G. Avaliação da atividade moluscicida do látex de três espécies de Euphorbia (Euphorbiaceae) sobre Leptinaria unilamellata D’Orbigny, 1835 (Gastropoda - Subulinidae). Revista Brasileira de Plantas Medicinais, v.12, n.1, p.90-95, 2010.

BARBOSA, A.F.; SALGADO, N.C.; COELHO, A.C.S.; MONTEIRO, J.C. Achatina fulica Bowdich, 1822, o "Caramujo Gigante Africano": história, biologia e controle de uma praga em expansão no Brasil (Mollusca, Gastropoda, Achatinoidea). Informativo da Sociedade Brasileira de Malacologia, v.33, n.140, p.4-5, 2002.

BARKER, G.M.; WATTS, C. Management of the invasive alien snail Cantareus aspersus on conservation land. Wellington: Department of Conservation; 2002.

BEIGUELMAN, B. Curso prático de bioestatística. $5^{\text {a }}$ ed. Ribeirão Preto: Funpec; 2002. 274p.

COLLEY, E.; FISCHER, M.L. Avaliação dos problemas enfrentados no manejo do caramujo gigante africano Achatina fulica (Gastropoda: Pulmonata) no Brasil. Zoologia, v.26, n.4, p.674-683, 2009.

COLLEY, E. Medidas de controle de Achatina fulica. In: FISCHER, M.L.; COSTA, L.C.M. (Eds.). O caramujo gigante africano Achatina fulica no Brasil. Curitiba: Champagnat Editora - PUCPR; 2010. p.203-229.

COWIE, R.H.; ROBINSON, D.G. Pathways of introduction of nonindigenous land and freshwater snails and slugs. In: RUIZ, G.; CARLTON, J.T. (Ed.) Invasive species: vectors and management strategies. Washington, D.C.: Island Press; 2003. p.93-122.

GOBBO-NETO, L.; LOPES, N.P. Plantas medicinais: fatores de influência no conteúdo de metabólitos secundários. Química Nova, v.30, p.374-381, 2007.

LEYTON, V.; HENDERSON, T.O.; MASCARA, D.; KAWANO, T. Atividade moluscicida de princípios ativos de folhas de Lycopersicon esculentum (Solanales, Solanaceae) em Biomphalaria glabrata (Gastropoda, Planorbidae). Iheringia: Série Zoologia, v.95, n.2, p.213-216, 2005.

MABBERLEY, D.J. The plant-book: a portable dictionary of the higher plants. $2^{\mathrm{a}}$ ed. Cambridge: Cambridge University Press; 1997. 858p.

MARSTON, A.; HOSTETTTMANN, K. Plant molluscicides. Phytochemistry, v.24, n.4, p.639-52, 1985.
MARSTON, A.; DUDAN, G.; GUPTA, M.P.; SOLIS, P.N.; CORREA, M.D.; HOSTETTMANN, K. Screening of Panamanian plants for molluscicidal activity. International Journal Pharmaceutics, v.34, p.15-18, 1996.

MEYER, W.M.; HAYES, K.A.; MEYER, A.L. Giant African snail, Achatina fulica, as a snail predator. America Malacological Bulletin, v.24, p.117-119, 2008.

NASCIMENTO, C.A.A.; ARÉVALO, E.; AFONSO-NETO, I.S.; ALMEIDA BESSA, E.C.A.; SOARES, G.L.G. Efeito do extrato aquoso de folhas de Allamanda cathartica L. (Apocynaceae) sobre Bradybaena similaris (Férussac, 1821) (Mollusca, Bradybaenidae) em condições de laboratório. Revista Brasileira de Zoociências, v.8, n. 1, p.77-82, 2006.

OLIVEIRA, F.; AKISUE, G. Fundamentos de Farmacobotânica. $2^{a}$ ed. São Paulo: Atheneu; 1997. 178p.

OLIVEIRA, F.Q.; GOBIRA, B.; GUIMARÃES, C.; BATISTA, J.; BARRETO, M.; SOUZA, M. Espécies vegetais indicadas na odontologia. Revista Brasileira de Farmacognosia, v. 17, p.466-476, 2007.

OLIVEIRA, R.A.; REIS, T.V.; SACRAMENTO, C.K.; DUARTE, L.P.; OLIVEIRA, F.F. Constituintes químicos voláteis de especiarias ricas em eugenol. Revista Brasileira de Farmacognosia, v. 19, n.3, p.771-775, 2008.

PEREIRA, J.P.; SOUZA, C.P.; MENDES, N.M. Propriedades moluscicidas da Euphorbia cotinifolia L. Revista Brasileira de Pesquisas Médicas e Biológicas, v.1 1, n.6, p.345-351, 1978.

PINHEIRO, L.; CORTEZ, D.A.G. Estudo fitoquímico e avaliação da atividade moluscicida da Kielmeyera variabilis Mart (Clusiaceae). Química Nova, v.26, n.2, p.157-160, 2003.

RAINA, V.K.; SRIVASTAVA, S.K.; AGGARWAL, K.K.; SYAMASUNDAR, K.V.; KUMAR, S. Essential oil composition of Syzygium aromaticum leaf from Little Andaman, India. Flavour Fragrance Journal, v. 16, p.334-336, 2001.

RAUT, K.; BARKER, G. Achatina fulica Bowdich and others Achatinidae pest in tropical agriculture. In: BARKER, G.M. (Ed.). Mollusks as croup pest. London, UK: CAB Publishing; 2002. p.55-114.

RUG, M.; RUPPEL, A. Toxic activities of the plant Jatropa curcas against intermediate snail hosts and larvae of schistosomes. Tropical Medicine \& International Health, v.5, n.6, p.423-430, 2000.

RUIZ, A.L.T.; MAGALHÃES, E.G.; MAGALHÃES, A.F.; FARIAS, A.D.; AMARAL, M.C.E.; SERRANO, D.R.; ZANOTTI-MAGALHÃES, E.M.; MAGALHÃES, L.A. Avaliação da atividade tóxica em Artemia salina e Biomphalaria glabrata de extratos de quatro espécies do gênero Eleocharis (Cyperaceae). Revista Brasileira de Farmacognosia, v.15, n.2, p.98-102, 2005.

SANTOS, A.B.S.; SILVA, T.F.B.; PAIVA, L.M.; SANTOS, A.C.; ALVESLIMA, E.A.L. Efeito fungitóxico do óleo de nim sobre Metarhizium anisopliae var. acridum e Metarhizium anisopliae var. anisopliae. Revista Caatinga, v.22, n.2, p.17-22, 2009. 
SIMIÃO, M.S.; FISCHER, M.L. Estimativa e inferência do método de controle do molusco exótico Achatina fulica Bowdich, 1822 (Stylommatophora; Achatinidae) em pontal do Paraná, litoral do Estado do Paraná. Cadernos de Biodiversidade, v.4, p.74-83, 2004.

SINGH, K.L; SINGH, D.K.; SINGH, V.K. Characterization of the molluscicidal activity of Bauhinia variegata AND Mimusops elengi plant extracts against the Fasciola vector Lymnaea acuminate. Revista do Instituto de Medicina Tropical de São Paulo, v.54, n.3, p. 135-140, 2012.

TELLES, H.M.S.; VAZ, J.F.; FONTES, L.O.R.; DOMINGOS, M.F. Registro de Achatina fulica Bowdich, 1822 (Mollusca, Gastropoda) no Brasil: caramujo hospedeiro intermediário da angiostrongilíase. Revista de Saúde Pública, v.31, n.3, p.310-312, 1997.

THIENGO, S.C.; FARACO, F.A.; SALGADO, N.C.; COWIE, R.H.; FERNANDEZ, M.A. Rapid spread of an invasive snail in South America: the giant African snail, Achatina fulica, in Brazil. Biological Invasions, v.9, n.6, p.1-10, 2007.

THIENGO, S.C.; FERNANDEZ, M.A.; TORRES, E.J.L.; COELHO, P.M.; LANFREDI, R.M. First record of a nematode Metastrongyloidea
(Aelurostrongylus abstrusus larvae) in Achatina (Lissachatina) fulica (Mollusca, Achatinidae) in Brazil. Journal of Invertebrate Pathology, v.98, p.34-39, 2008.

TSAO, R.; YU, Q. Nematicidal activity of monoterpenoid compounds against economically important nematodes in agriculture. Journal of Essential Oil Research, v. 12, p.350-354, 2000.

YADAV, R.P.; SINGH, A. Efficacy of Euphorbia hirta latex as plant derived molluscicides against freshwater snails. Revista do Instituto de Medicina Tropical de São Paulo, v.53, n.2, p.101106, 2011.

ZANI, C.L.; MARSTON, A.; HAMBURGER, M.; HOSTETTMANN, K. Molluscicidal miliamines from Euphorbia milii var. hislopii. Phytochemistry, v.34, n.1, p.89-95, 1993.

ZANOL, J.; FERNANDEZ, M.A.; OLIVEIRA, A.P.M.; RUSSO, C.A.M.; THIENGO, S.C. O caramujo exótico invasor Achatina fulica (Stylommatophora, Mollusca) no Estado do Rio de Janeiro (Brasil): situação atual. Biota Neotropica, v.10, n.3, p.447-451, 2010. 